\title{
Safety, tolerability and efficacy of the glutaminyl cyclase inhibitor PQ912 in Alzheimer's disease: results of a randomized, double-blind, placebo-controlled phase $2 \mathrm{a}$ study
}

Philip Scheltens ${ }^{1 *}$ (D), Merja Hallikainen², Timo Grimmer ${ }^{3}$, Thomas Duning ${ }^{4}$, Alida A Gouw ${ }^{1,5}$, Charlotte E Teunissen ${ }^{6}$, Alle Meije Wink ${ }^{7}$, Paul Maruff ${ }^{8}$, John Harrison ${ }^{1,9}$, Caroline M van Baal ${ }^{10}$, Suzanne Bruins ${ }^{11}$, Inge Lues ${ }^{11}$ and Niels D Prins ${ }^{1,12}$

\begin{abstract}
Background: PQ912 is an inhibitor of the glutaminyl cyclase enzyme that plays a central role in the formation of synaptotoxic pyroglutamate-A-beta oligomers. We report on the first clinical study with PQ912 in subjects with biomarker-proven Alzheimer's disease (AD). The aim was to determine the maximal tolerated dose, target occupancy and treatment-related pharmacodynamic effects. The exploratory efficacy readouts selected were tailored to the patient population with early AD. The therapeutic approach focuses on synaptic dysfunction as captured by various measures such as electroencephalography (EEG), synaptic biomarkers and sensitive cognitive tests.
\end{abstract}

Methods: This was a randomized, double-blind, placebo-controlled trial evaluating the safety, tolerability and efficacy of PQ912 800 mg twice daily (bid) for 12 weeks in subjects with mild cognitive impairment or mild dementia due to AD. The 120 enrolled subjects were treatment-naive at the start of the study, had confirmed AD biomarkers in their cerebrospinal fluid at screening and had a Mini Mental State Examination score between 21 and 30. After 1 week of treatment with $400 \mathrm{mg}$ bid, patients were up-titrated to $800 \mathrm{mg}$ bid for 11 weeks. Patients were randomized 1:1 to either PQ912 or placebo. The primary composite endpoints were to assess safety and tolerability based on the number of patients who discontinued due to (serious) adverse events (safety), and based on dose adjustment during the treatment period and/or nonadherence to randomized treatment (tolerability). All randomized subjects who took at least one dose of the study treatment or placebo were used for safety analyses.

(Continued on next page)

\footnotetext{
* Correspondence: p.scheltens@vumc.nl

${ }^{1}$ Alzheimer Center and Department of Neurology, Amsterdam Neuroscience,

VU University Medical Center, Amsterdam, The Netherlands

Full list of author information is available at the end of the article
}

(c) The Author(s). 2018 Open Access This article is distributed under the terms of the Creative Commons Attribution 4.0 International License (http://creativecommons.org/licenses/by/4.0/), which permits unrestricted use, distribution, and reproduction in any medium, provided you give appropriate credit to the original author(s) and the source, provide a link to the Creative Commons license, and indicate if changes were made. The Creative Commons Public Domain Dedication waiver (http://creativecommons.org/publicdomain/zero/1.0/) applies to the data made available in this article, unless otherwise stated. 
(Continued from previous page)

Results: There was no significant difference between treatments in the number of subjects with (serious) adverse events, although there were slightly more patients with a serious adverse event in the PQ912 group compared to placebo. More subjects treated with PQ912 discontinued treatment due to adverse events, mostly related to gastrointestinal and skin/subcutaneous tissue disorders. PQ912 treatment resulted in a significant reduction in glutaminyl cyclase activity, which resulted in an average target occupancy of $>90 \%$. A significant reduction of theta power in the EEG frequency analysis and a significant improvement in the One Back test of our Neuropsychological Test Battery was observed. The exploratory biomarker readouts, neurogranin for synaptic toxicity and YKL-40 as a marker of inflammation, appear to be sensitive enough to serve as efficacy markers in the next phase $2 b$ study.

Conclusions: The maximal tolerated dose of PQ912 has been identified and the results support future studies at still lower doses reaching $>50 \%$ target occupancy, a longer up-titration phase to potentially induce adaptation and longer treatment periods to confirm the early signals of efficacy as seen in this study.

Trial registration: Clinicaltrials.gov, NCT 02389413. Registered on 17 March 2015.

Keywords: Alzheimer's disease, Glutaminyl cyclase inhibitor, PQ912, Phase 2a

\section{Background}

Alzheimer's disease (AD) is the most prevalent form of dementia, affecting more than 40 million people worldwide with prevalence expected to double by 2030 [1]. At present, only symptomatic treatment is available, and there is a strong need for disease-modifying treatments. Although the etiology of AD is not understood completely, there is agreement that beta-amyloid $(A \beta)$ plaques and tau tangles are central to its biology [2-5]. Different $\mathrm{A} \beta$ species can form soluble oligomers of varying size. However, in contrast to insoluble $A \beta$ plaques, soluble $A \beta$ oligomers are accepted as the primary source of toxicity because they lead to synaptic impairment, reduce spike numbers, impair neuronal connectivity and result in tau-dependent neurodegeneration over the course of the disease $[6,7]$. Consequently, $A \beta$ oligomers may be an important therapeutic target for halting AD. Studies into the molecular events of $\mathrm{A} \beta$ oligomer composition and formation show the importance of the enzyme glutaminyl cyclase $(\mathrm{QC})$ in these processes [8]. Increasing full-length $\mathrm{A} \beta_{1-40}$ or $\mathrm{A} \beta_{1-42}$ levels early in $\mathrm{AD}$ leads to activation of various peptidases (e.g., neuroendopeptidase), which in turn cause truncated versions of $\mathrm{A} \beta$ with an $\mathrm{N}$-terminal glutamate at positions 3 and 11. These truncated versions are then modified by glutamate cyclization to pyroglutamate (pGlu) by QC, as illustrated in Fig. 1. The pGlu-A $\beta$-seeded $A \beta$ oligomers possess a very high synaptotoxic, neurotoxic and proinflammatory potential in in-vitro and animal models $[8,9]$. pGlu-A $\beta$-seeded oligomers strongly suppressed LTP in brain slices, an indicator of synaptic impairment [8]. In a translational study in an AD animal model, PQ912 significantly rescued impaired spatial learning and memory at a dose which achieved between 50 and 70\% QC inhibition in the spinal fluid [9]. pGlu-A $\beta$ has been shown to induce changes in the secondary and tertiary structure of oligomers and these changes most likely are responsible for the increased synaptotoxic propensity of pGlu-A $\beta$-containing aggregates $[8,10,11]$. The mechanisms of synaptotoxicity of pGlu-A $\beta$ oligomers have been shown to be different compared to $A \beta$ oligomers [12].

The discovery of pGlu-A $\beta$ pathobiology provides a foundation for a new therapeutic approach in AD where inhibition of the activity of QC could reduce the production of pGlu-A $\beta$ in the brain. This could subsequently alleviate the acute and chronic neurotoxic effects of pGlu-A $\beta$ oligomers. Consequently, long-term inhibition of QC activity would allow synaptic regeneration and reduced neuronal death rates which should manifest clinically as a reduction in cognitive decline and lower rates of clinical disease progression. PQ912, an inhibitor of $\mathrm{QC}$, has undergone rigorous preclinical and clinical investigations including in-vitro and in-vivo animal and phase 1 studies $[9,13]$ and is a first-in-class QC inhibitor in clinical development. This phase $2 \mathrm{a}$ study had the objective to investigate the highest PQ912 dose that was used in an earlier phase 1 study of the same compound [13], to evaluate safety and tolerability signals in

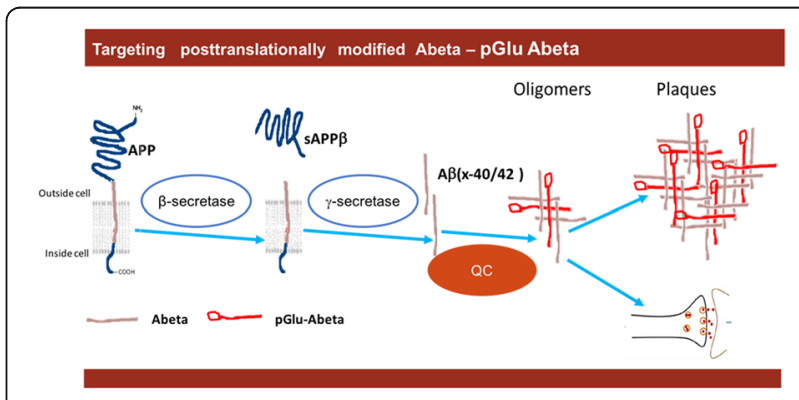

Fig. 1 Schematic drawing of $Q C$ inhibitor approach. pGlu-A $\beta$ is posttranslationally formed by $Q C$ from $N$-terminally truncated $A \beta$ versions carrying a glutamate at position N3 and N11. pGlu-A $\beta$ seeds $A \beta$ oligomers which are highly synaptotoxic and neurotoxic. $A \beta$ amyloid beta, pGlu pyroglutamate, QC glutaminyl cyclase, sAPP $\beta$ soluble amyloid precursor protein beta 
treatment-naïve subjects with mild cognitive impairment (MCI) due to $\mathrm{AD}$ or mild dementia due to $\mathrm{AD}$ after 12 weeks of treatment and also to evaluate the usefulness of various efficacy measurements for future studies using longer treatment durations.

\section{Methods}

\section{Study design}

This was a multicenter, randomized, double-blind, placebo-controlled, parallel-group safety and tolerability phase 2 study of PQ912. Efficacy was assessed in an exploratory manner.

Subjects satisfying all selection criteria at screening were randomly assigned in a 1:1 ratio to receive either PQ912 or placebo for 12 weeks. Dosage of PQ912 was $400 \mathrm{mg}$ bid in the first week, followed by $800 \mathrm{mg}$ bid for 11 weeks. During the study, visits to the study site occurred at baseline, after 3, 6 and 12 weeks on treatment and 28 days after the end of treatment.

Procedures at screening included documentation of medical history, physical and neurological examination, assessments of vital signs, ECG, EEG, MMSE, Geriatric Depression Scale, Neuropsychological Test Battery and MRI including resting state functional (RSf)MRI. Blood and urine samples were collected for blood chemistry, hematology, parameters related to QC substrates (thyroid-stimulating hormone (TSH), total triiodothyronine (T3), free prohormone thyroxine (T4), testosterone) and for ApoE genotyping. A CSF sample was collected for biomarker assessment.

The MMSE and Neuropsychological Test Battery were repeated at baseline and end of treatment (EOT); CSF sampling, MRI, ECG and EEG were repeated at EOT only. Physical and neurological examination, vital signs and blood and urine sampling were repeated at each study visit. (S)AEs and concomitant medication were reported throughout the study.

At baseline and EOT, a 20-min resting-state EEG was recorded against a common reference at 21 electrode positions of the 10-20 system. Patients were seated in a slightly reclined chair in a sound-attenuated but fully lit room and were instructed to keep their eyes closed and stay awake. EEG technicians monitored the recording carefully and alerted the patients by sound stimuli at the first signs of drowsiness.

Central analysis/reading was done for clinical laboratory, genotyping, MRI images, EEG data and biomarkers. Cogstate performed central monitoring on the computerized test data.

\section{Subjects}

A total of 120 treatment-naïve men $(n=56)$ and women $(n=64)$, aged 51-85 years, with a diagnosis of mild cognitive impairment due to $\mathrm{AD}$ or mild dementia due to $\mathrm{AD}$, according to the Alzheimer Association-National Institute on Aging criteria $[14,15]$, were included in the study. Other inclusion criteria were a Mini Mental State Examination (MMSE) score between 21 and 30, and a brain magnetic resonance imaging (MRI) scan consistent with the diagnosis of the disease. In addition, subjects had to show a positive $\mathrm{AD}$ biomarker signature at screening.

\section{Safety and tolerability endpoints}

Two primary safety and tolerability composite endpoints were defined. Safety was based on: discontinuation of subject due to a serious adverse event (SAE); or discontinuation of subject due to an adverse event (AE) with severity $\geq 3$ according to the Common Terminology Criteria for Adverse Events (CTCAE); or discontinuation of subject due to an extreme laboratory parameter. Tolerability was based on: dose adjustment during the treatment period; and/or nonadherence to randomized treatment.

Secondary tolerability endpoints were: number of SAEs and AEs with severity $\geq 3$ according to the CTCAE; the time to first appearance of AEs; time to dose adjustment; time to nonadherence; and a composite of time to dose adjustment, nonadherence, discontinuation due to SAE, AE with severity $\geq 3$ according to the CTCAE or extreme laboratory parameters.

Other safety measures were AEs, vital signs, ECG measurements, clinical laboratory tests, changes on brain MRI scans, and physical and neurologic examinations.

\section{Efficacy endpoints}

The following endpoints were defined for exploratory efficacy.

- CSF biomarkers: diagnostic biomarkers $A \beta_{1-42}$, tau and P-tau (Innotest, Fujirebio); and exploratory biomarkers QC activity, primary target of inhibitor (Evotec AG), neurogranin and beta secretase I (ADxNeurosciences/Euroimmun), Contactin 2 ( $\mathrm{R}$ and $\mathrm{D}$ systems, duoset), neurofilament light chain (Uman diagnostics) and Chitinase-3-like protein 1 (CHI3L1 = YKL-40) (Quidel Corporation).

- Neuronal oscillatory activity and network anaysis as measured by EEG: mean peak frequency in the parieto-occipital region, global relative alpha and theta power $(8-13 \mathrm{~Hz}$ and $4-8 \mathrm{~Hz}$, respectively), mean global phase lag index in the alpha band and network topology measures in the alpha band, based on the minimum spanning tree of the full network - mean phase lag index, leaf fraction and tree hierarchy in the alpha band.

- MRI: normalized brain volume at screening and percentage brain volume change at EOT.

- RSfMRI: mean $z$-statistic default mode network (DMN), mean eigenvector centrality values and mean path length and clustering coefficient. 
- Cognition: episodic memory

(average of standardized scores of the OCL Test, ISLT and ISLT-delayed recall), executive function (average of standardized scores of LFT, CFT and One Back Test), attention (average of standardized scores of Detection Test and Identification Test), overall cognition (average of standardized scores of all cognitive measures; scores from at least six tests needed for calculation) and MMSE.

\section{QC activity and inhibition}

QC activity in CSF was measured by Evotec AG (Hamburg) and PQ912 by Swiss Bioquant (Basel) as described by Lues et al. [13].

In-vivo QC target occupancy (TO) was calculated from PQ912 CSF levels for CSF samples collected within $24 \mathrm{~h}$ after the last compound intake at the EOT visit by applying the following formula:

$$
\mathrm{TO}(\%)=100 \times C /\left(K_{\mathrm{i}}+C\right)
$$

where $\mathrm{TO}=$ target occupancy $(\%), K_{\mathrm{i}}=$ inhibitory constant of PQ912 (25 nM) and $C=$ measured CSF concentration of PQ912 Additional file 2: Table S1.

\section{Statistical analysis}

No formal sample size calculation was conducted, since this was not possible in the absence of any clinically relevant incidence of adverse events or drug discontinuations in a previously conducted comprehensive phase 1 study for PQ912. In order to gain sufficient safety and tolerance information, we planned a sample size of 110 subjects (55 per group) which allows an exposure of 10 patient-years in each study arm (55 subjects $\times 3$ months minus potential early discontinuation) in this first in-patient study.

The effect of treatment on the primary composite endpoints was evaluated using Fisher's exact tests $\left(\alpha_{(2-\text {-sided }}\right)$ $=0.05)$. The relative risk $(R R)$ and risk difference $(R D)$ were reported with a 95\% confidence interval (CI). Kaplan-Meier plots and log-rank tests were used for time to event outcomes (first appearance of AEs, dose adjustment, nonadherence or a composite of dose adjustment, nonadherence, discontinuation due to SAE and AE with severity $\geq 3$ according to CTCAE or extreme laboratory parameters). Descriptive statistics were used for other safety measures.

Analysis of the exploratory efficacy parameters was performed in three distinct populations: intention to treat (ITT), defined as all randomized subjects who took at least one dose of the study treatment or placebo; modified ITT (mITT), defined as the ITT population excluding subjects who started using acetylcholinesterase inhibitor during treatment period; and per protocol (PP), defined as all randomized subjects without major protocol deviations. Analysis of the cerebrospinal fluid (CSF) biomarker endpoints was conducted in the PP population because in this population the timing of last treatment and sampling occurred within a 24-h window. Efficacy endpoints were first tested for normality, and transformed if necessary. All CSF biomarkers were $\log _{10}$ transformed before statistical analysis, as well as two EEG parameters and one RSfMRI parameter. Single imputation was performed for ITT and mITT analyses. $\alpha_{(2-\text { sided })}=0.05$ was used without adjustment for multiple comparisons.

An ANCOVA with treatment, gender, ApoE and country (stratification) as factors and time between screening and baseline, baseline measure of the endpoint and age as covariates was performed for cognitive, CSF and neuronal network endpoints. Cohen's $D(d)$ treatment effect sizes and differences between treatment groups, corrected for the previously mentioned covariates ( $\delta$, with $95 \% \mathrm{CI}$ ), were reported for these parameters. Due to many observations below the limit of quantification, data for pGlu-A $\beta$ monomer and pGlu-A $\beta$ oligomer were dichotomized (yes or no quantifiable) and analyzed by Mantel-Haenszel chi-square statistics.

\section{Results \\ Demographics}

Out of a total of 238 subjects who were screened, 120 subjects were enrolled in the study and randomized to treatment with PQ912 $(n=60)$ or placebo $(n=60) ; 115$ subjects completed the end of treatment (EOT) visit (55 in the PQ912 group and 60 in the placebo group). These numbers are also shown in the CONSORT flow diagram (Fig. 2). Five subjects treated with $\mathrm{PQ} 912$ withdrew their consent and did not complete the EOT visit. The subject disposition for each study group is presented in Table 1 . The only difference between groups at baseline was that the PQ912 group contained more women (60\%) than the placebo group (47\%). Carriage of the apolipoprotein E (APoE) E4 allele was as expected and equivalent in both groups (63\% in the PQ912 group and 72\% in the placebo group).

\section{Safety and tolerability}

There were slightly more patients with a serious adverse event (SAE) in the PQ912 group compared to placebo, although statistically not significant. In the PQ912 group, eight subjects reported $13 \mathrm{SAEs}$ and in the placebo group, five subjects reported five SAEs. The SAEs reported were heterogeneous in nature and showed no pattern pointing to a specific effect of PQ912. Six subjects in the PQ912 group discontinued treatment due to the SAEs and none in the placebo group.

The number of subjects with treatment emergent adverse events (TEAEs) was not different between study groups, although the PQ912-treated subjects reported 


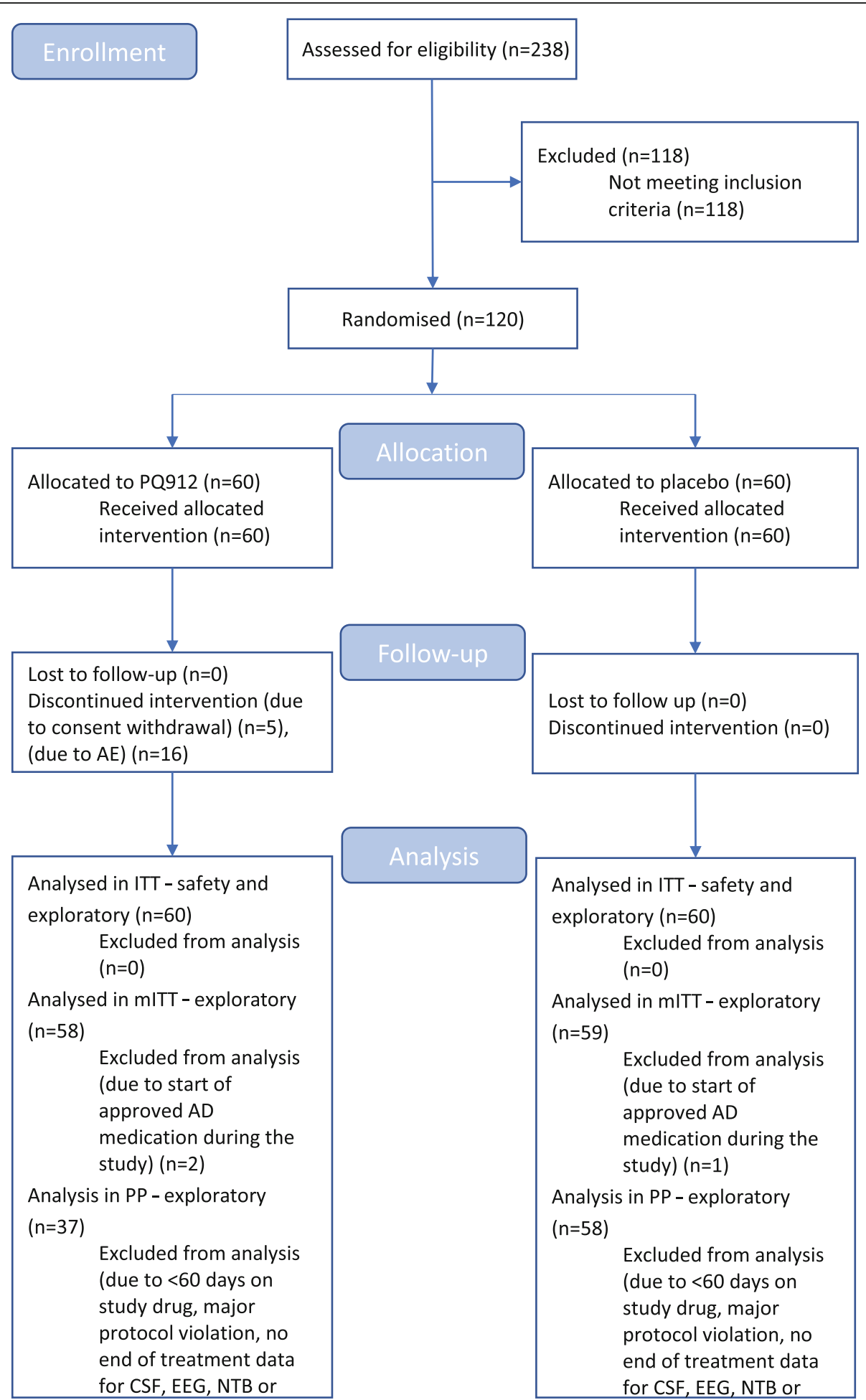

Fig. 2 CONSORT flow diagram. AD Alzheimer's disease, AE adverse event, CSF cerebrospinal fluid, EEG electroencephalography, ITT intention to treat, mITT modified intention to treat, MRI magnetic resonance imaging, NTB Neuropsychological Test Battery, PP per protocol

slightly more TEAEs. In the PQ912 group, 45 subjects reported 135 TEAEs, while 40 subjects in the placebo group reported 103 TEAEs. The majority of AEs were classified as mild or moderate in severity. Subjects in the PQ912 group reported AEs most frequently in the system organ class (SOC) categories of gastrointestinal 
Table 1 Demographics and other baseline characteristics

\begin{tabular}{llll}
\hline & Statistic & $\begin{array}{l}\text { Placebo } \\
(N=60)\end{array}$ & $\begin{array}{l}\text { PQ912 } \\
(N=60)\end{array}$ \\
\hline Age (years) & Mean (SD) & $72(7)$ & $70.8(8)$ \\
& & & \\
Female & Sex & & $36(60)$ \\
Male & $n(\%)$ & $28(47)$ & $24(40)$ \\
MMSE & $n(\%)$ & $32(53)$ & $25.2(3)$ \\
& Mean (SD) & $24.8(3)$ & \\
E4 & APoE & & $38(63)$ \\
\hline
\end{tabular}

APOE apolipoprotein E, MMSE Mini Mental State Examination, $N$ number of subjects in population, $n$ number of subjects, $S D$ standard deviation

disorders $(n=21)$, infections and infestations $(n=17)$, and skin and subcutaneous tissue disorders $(n=15)$. The most frequently reported TEAEs in the placebo group were in the SOC categories of gastrointestinal disorders $(n=12)$ and infections and infestations $(n=17)$. Five placebo subjects reported skin and subcutaneous tissue disorders (Table 2).

The greatest differences between incidences of TEAEs were observed in the SOC categories of gastrointestinal disorders and skin and subcutaneous tissue disorders. Within these two categories, subjects in the PQ912 group reported mostly nausea, diarrhea and constipation, and rash and urticaria. Subjects in the placebo group reported mostly nausea, abdominal pain upper and rash in these categories. AEs in the SOC category of infections and infestations were mostly viral upper respiratory tract infection and upper respiratory tract infection, and showed no difference between treatment arms (Table 2). The onset of TEAEs in the SOC of skin and subcutaneous tissue disorders occurred mostly in week 2 to week 8 (Fig. 3a).

In both study groups, a reduction in the prescribed dose from $800 \mathrm{mg}$ to $400 \mathrm{mg}$ twice daily (bid) was observed in five subjects. In the PQ912 group, 26 subjects $(43.3 \%)$ met the criteria for nonadherence to study treatment; 20 of them discontinued due to AEs, five continued treatment and one withdrew consent. Four of the 20 subjects who discontinued treatment also withdrew consent and did not complete the study. In the placebo group, two subjects (3.3\%) qualified for nonadherence to study treatment, but did not discontinue the study. Time to nonadherence was reached significantly earlier for subjects in the PQ912 group compared to placebo $(P<0.001)$. The majority of treatment discontinuations were observed between treatment weeks 2 and 8 (Fig. 3b).

Prospectively defined composite endpoints for safety and for tolerability were significantly different between the PQ912 and placebo groups $(P=0.027$ for safety and $P<0.001$ for tolerability, Table 3$)$.
Table 2 Treatment emergent adverse events summary and distribution by Medical Dictionary for Regulatory Activities (MedDRA) system organ classes (SOC) (with $\geq 3$ subjects reporting in total)

\begin{tabular}{lll}
\hline TEAE summary & $\begin{array}{l}\text { Placebo } \\
(N=60)\end{array}$ & $\begin{array}{l}\text { PQ912 } \\
(N=60)\end{array}$ \\
Subjects with any TEAE & $40(67)$ & $45(75)$ \\
Subjects who discontinued due to TEAE & & $20(33)$ \\
Subjects with TEAE by severity & 0 & \\
$\quad$ Mild & & \\
$\quad$ Moderate & $27(45)$ & $17(28)$ \\
Severe & $12(20)$ & $20(33)$ \\
Number of TEAEs & $1(2)$ & $8(13)$ \\
Subjects with serious TEAEs & 103 & 135 \\
Number of SAEs & $3(5)$ & $8(13)$ \\
Number of serious TEAEs & 5 & 13 \\
Deaths & 3 & 13 \\
\end{tabular}

MedDRA SOC - preferred term

Blood and lymphatic system disorders $\quad 1 \quad 2$

Cardiac disorders

2

Gastrointestinal disorders

22

Abdominal pain upper

Constipation

Diarrhea

Dyspepsia

Nausea

Vomiting

General disorders and administration site conditions $6 \quad 5$

Hepatobiliary disorders $\quad 0 \quad 3$

$\begin{array}{lll}\text { Infections and infestations } & 17 & 17\end{array}$

Injury, poisoning and procedural complications $\quad 5 \quad 6$

Investigations $\quad 5 \quad 8$

$\begin{array}{lll}\text { Metabolism and nutrition disorders } & 2 & 7\end{array}$

Musculoskeletal and connective tissue disorders $\quad 8 \quad 5$

$\begin{array}{lll}\text { Nervous system disorders } & 9 & 7\end{array}$

$\begin{array}{lll}\text { Psychiatric disorders } & 3 & 4\end{array}$

Renal and urinary disorders $\quad 1 \quad 3$

Skin and subcutaneous tissue disorders $\quad 5 \quad 15$

Rash

Rash generalized

Rash maculopapular

Urticaria

Vascular disorders

(1)
8 5

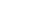

(7)

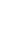

$$
7
$$

(1)

Data presented as $n(\%)$ or $n$

$N$ number of subjects in population, $n$ number of subjects, $S A E$ serious adverse event, SOC system organ class, TEAE treatment emergent adverse event

${ }^{a}$ These subjects may still have completed end-of-study assessment 

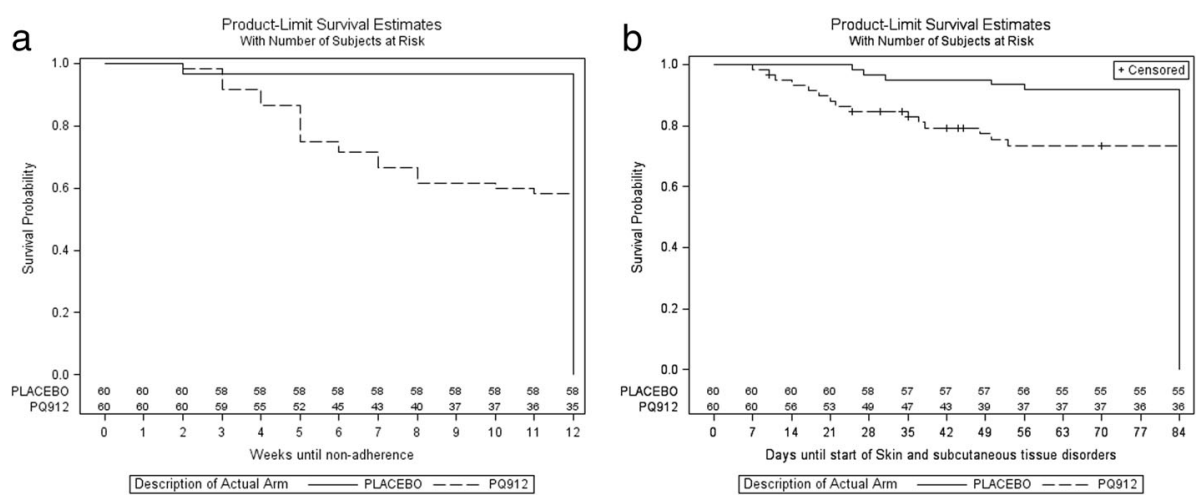

Fig. 3 a Time to nonadherence. Intent to treat population. $\mathbf{b}$ Time to start of TEAEs of skin and subcutaneous tissues disorders . Intent to treat population

Overall, we found no clinically relevant differences between the PQ912 and placebo groups in changes over time in any of the clinical chemistry and hematology parameters or parameters related to physiological QC substrates. We observed shifts from normal to out of normal

Table 3 Safety and tolerability endpoints

\begin{tabular}{|c|c|c|c|}
\hline & $\begin{array}{l}\text { Placebo } \\
(N=60), \\
n(\%)\end{array}$ & $\begin{array}{l}\mathrm{PQ} 912 \\
(N=60) \\
n(\%)\end{array}$ & $P$ \\
\hline \multicolumn{4}{|l|}{ Safety } \\
\hline Composite safety & $0(0.0)$ & $6(10.0)$ & 0.027 \\
\hline Discontinuation of subject due to SAE & $0(0.0)$ & $6(10.0)$ & 0.027 \\
\hline $\begin{array}{l}\text { Discontinuation of subject due to } A E \\
\text { with severity } \geq 3 \text { according to CTCAE }\end{array}$ & $0(0.0)$ & $6(10.0)$ & 0.027 \\
\hline $\begin{array}{l}\text { Discontinuation of subject due to } \\
\text { extreme safety laboratory parameters }\end{array}$ & $0(0.0)$ & $0(0.0)$ & - \\
\hline Number of subjects with SAES & $5(8.3)^{d}$ & $8(13.3)$ & 0.558 \\
\hline $\begin{array}{l}\text { Number of subjects with AEs with } \\
\text { severity } \geq 3 \text { according to CTCAE }\end{array}$ & $3(5.0)^{e}$ & $8(13.3)$ & 0.204 \\
\hline \multicolumn{4}{|l|}{ Tolerability } \\
\hline Composite tolerability & $6(10.0)$ & $27(45.0)$ & $<.001$ \\
\hline $\begin{array}{l}\text { Dose adjustment during treatment } \\
\text { period }^{\mathrm{a}}\end{array}$ & $5(8.3)$ & $5(8.3)$ & 1.000 \\
\hline Nonadherence to randomized treatment ${ }^{\mathrm{b}}$ & $2(3.3)$ & $26(43.3)$ & $<.001$ \\
\hline Discontinued due to related $\mathrm{AE}^{\mathrm{C}}$ & $0(0.0)$ & $19(31.7)$ & $<.001$ \\
\hline Discontinued due to nonrelated $\mathrm{AE}$ & $0(0.0)$ & $1(1.7)$ & 1.000 \\
\hline Withdrawal of consent by subject & $0(0.0)$ & $1(1.7)$ & 1.000 \\
\hline Continued & $2(3.3)$ & $5(8.3)$ & 0.439 \\
\hline
\end{tabular}

CTCAE common terminology criteria for adverse events, $N$ number of subjects in population, $n$ number of subjects, (S)AE (serious) adverse event

${ }^{a}$ Dose adjustment defined as reduction of dose from $800 \mathrm{mg}$ bid to $400 \mathrm{mg}$ bid, as allowed within the study protocol

bNonadherence defined as: using $<75 \%$ of prescribed dose in 4 consecutive weeks including at least 1 week with less than $50 \%$; or 3 or more consecutive days in total or 7 days of interrupted use during the full 12 weeks. Subjects could qualify for more than one criterion, but were listed once; reason ranked for relevance for tolerance

' Includes possibly related, probably related and related according to investigator ${ }^{\mathrm{d}}$ Treatment emergent in three subjects

${ }^{\mathrm{e}}$ Treatment emergent in one subject laboratory range values more frequently in the PQ912 group for hemoglobin, hematocrit and alkaline phosphatase, although these values normalized toward EOT and became normal by the follow-up assessment. One subject showed a clinically significant increase in transaminases at EOT, but this did not meet Hy's law criteria and values normalized after discontinuation of treatment with PQ912. As thyrotropin-releasing hormone (TRH) and gonadotropin-releasing hormone $(\mathrm{GnRH})$ are substrates of $\mathrm{QC}$, the downstream hormones thyroid-stimulating hormone (TSH), T3, T4 and testosterone were determined in blood and were not different between the PQ912 and placebo groups.

PQ912 treatment had no noticeable effect on vital signs and ECG, and no abnormal findings were noted in physical and neurological examinations and in brain magnetic resonance imaging (MRI) scans.

\section{Efficacy}

In total, 120 subjects were randomized to PQ912 treatment or placebo $\left(\mathrm{ITT} / \mathrm{mITT} / \mathrm{PP}\right.$ populations: $N_{\mathrm{PQ} 912}=$ $60 / 58 / 37, N_{\text {placebo }}=60 / 59 / 58$ ). All measures were prospectively defined in the SAP which was finalized before database lock. Additional file 1 summarizes the mean values and standard deviations of the efficacy measures at baseline and end of treatment and for the changes between end of treatment and baseline with 95\% confidence intervals. The effect sizes as expressed by Cohen's $D$ for those measures especially relevant to the concept are separately presented in Table 4.

\section{CSF biomarkers}

QC activity The mean QC activity was $118.5 \mathrm{mU} / \mathrm{L}$ (SD 33.1, $n=26$ ) at baseline and decreased to $46.0 \mathrm{mU} / \mathrm{L}$ (SD $24.3, n=26)$ at EOT $(P<0.001, d=1.66$, PP population). In the placebo group, the $\mathrm{QC}$ activity levels 
Table 4 Cohen's $d$ effect size for significant efficacy parameters

\begin{tabular}{llllll}
\hline Domain & Parameter & ITT population & mITT population & PP population & Effect size \\
\hline CSF & QC activity & $1.25^{* * *}$ & $1.28^{* * *}$ & $1.66^{* * *}$ & $\downarrow$ \\
EEG & Relative theta power & $0.29^{* *}$ & $0.32^{* * *}$ & $0.37^{* *}$ & $\downarrow$ large \\
NTB & One Back Test & $0.23^{*}$ & $0.23^{\dagger}$ & 0.20 & $\downarrow$ small-moderate \\
CSF & Neurogranin & $0.16^{\dagger}$ & $0.20^{*}$ & 0.12 & $\downarrow$ small \\
& YKL-40 & $0.16^{\dagger}$ & $0.16^{\dagger}$ & $0.20^{*}$ & $\downarrow$ small \\
& & & & small \\
\hline
\end{tabular}

CSF cerebrospinal fluid, EEG electroencephalogram, ITT intent to treat, $m / T T$ modified ITT, NTB Neuropsychological Test Battery, PP per protocol, QC glutaminyl cyclase

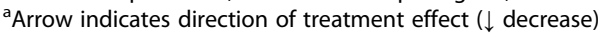

${ }^{+} 0.05<p \leq 0.10$

$* 0.01<p \leq 0.05$

${ }^{* *} 0.001<p \leq 0.01$

${ }^{* * *} p \leq 0.001$

remained constant (mean level at baseline $121.5 \mathrm{mU} / \mathrm{L}$, SD 31.5; at EOT 121.4 mU/L, SD 32.8, $n=41$ ) (Fig. 4a).

With the exception of one outlier with a low target occupancy (TO) of $2.1 \%$, the estimated median TO based on calculation from measured levels of PQ912 in CSF was $93.2 \%$ (interquartile range $89.0-94.6 \%$ ) (Fig. 4b).

YKL-40 The effect of PQ912 on change in the level of YKL-40 (a marker of glia activation) from baseline to EOT was statistically significant in the PP population $(P=$ $0.025, d=0.20$ ), indicating a stronger decrease in the PQ912 group compared to placebo. The mean of $344.2 \mathrm{pg} / \mathrm{ml}$ (SD 150.3) at baseline decreased to $324.3 \mathrm{pg} /$ $\mathrm{ml}(\mathrm{SD} 125.8)$ at EOT in the PQ912 group $(n=26)$, while in the placebo group there was no change: the mean was $386.5 \mathrm{pg} / \mathrm{ml} \mathrm{(SD} \mathrm{127.5)} \mathrm{at} \mathrm{baseline} \mathrm{and}$ $381.6 \mathrm{pg} / \mathrm{ml}$ (SD 142.9) at EOT $(n=41)$. The decrease of the means was $20 \mathrm{pg} / \mathrm{ml}(6 \%)$ in the PQ912 group and $5 \mathrm{pg} / \mathrm{ml}(1 \%)$ in the placebo group.

Neurogranin While neurogranin levels decreased in the PQ912 group this decrease was not consistently statistically significant different between study groups. In the mITT population there was a small, significant effect $(P$ $=0.036, d=0.20$ ), while in the PP and ITT populations changes in mean neurogranin levels were not significantly different, although there was a trend to decrease in the treatment group. In the PP population, the mean of $451.0 \mathrm{pg} / \mathrm{ml} \mathrm{(SD} \mathrm{193.4)} \mathrm{at} \mathrm{baseline} \mathrm{decreased} \mathrm{to}$ $431.8 \mathrm{pg} / \mathrm{ml}$ (SD 191.9) at EOT in the PQ912 group (n $=26$ ), while in the placebo group the mean was 477.6 (SD 188.1) $\mathrm{pg} / \mathrm{ml}$ at baseline and 468.4 $\mathrm{pg} / \mathrm{ml}$ (SD 192.4) at EOT $(n=41)$. The decrease of the means was $19 \mathrm{pg} /$ $\mathrm{ml}(4 \%)$ in the treatment arm and $10 \mathrm{pg} / \mathrm{ml}(2 \%)$ in the placebo group. ApoE had a statistically significant effect on neurogranin $(P=0.011, P=0.008$ and $P=0.025$ for ITT, mITT and PP populations, respectively), showing higher neurogranin values for E4 carriers in both study groups.
Diagnostic ( $\mathrm{A} \beta_{1-42}$, tau or P-tau levels) and other biomarkers (neurofilament light chain, beta secretase-I and Contactin-2) in the CSF did not change significantly across the 12-week period for both study groups.

\section{Neuronal oscillatory activity and functional network endpoints}

Spectral analyses of the oscillatory electroencephalography (EEG) activity showed a difference in mean change in global relative theta power $(4-8 \mathrm{~Hz})$ in the PQ912 group compared to placebo in the ITT $(P=0.002, d=0.29)$, mITT $(P<0.001, d=0.32)$ and PP $(P=0.002, d=0.37)$ populations. In the placebo group, the baseline global relative theta power increased from mean 0.1647 (SD 0.0794) to 0.1762 (SD 0.0819) at EOT (ITT population), whereas it decreased slightly in the PQ912 group from mean 0.1763 (SD 0.0976) to 0.1695 (SD 0.0881) at EOT (ITT population).

Global functional connectivity, as measured with the phase lag index [16] in the alpha band, and measures reflecting functional network topology (leaf fraction, mean phase lag index and tree hierarchy of the network, represented by its minimum spanning tree, an unbiased subnetwork of the full functional network $[17,18]$ ) did not show differences between treatment groups. Alpha band leaf fraction and tree hierarchy were influenced significantly by the ApoE4 allele (higher values in E4 carriers for alpha band leaf fraction, lower values in E4 carriers for tree hierarchy) with $P=0.009$ for alpha band leaf fraction and $P=$ 0.001 for tree hierarchy, respectively, in each of the ITT, mITT and PP populations.

PQ912 treatment had no effect on brain volume. Analyses of resting state functional (RSf)MRI endpoints showed no PQ912 treatment effects except for a statistically significant effect with lower EOT values in the PQ912 group compared to the placebo group observed in the mean eigenvector centrality of the cuneus and lateral occipital regions (posterior) in the ITT $(P=0.005, d$ $=0.58)$, $\operatorname{mITT}(P=0.003, d=0.60)$ and PP $(P=0.132$ and $d=0.39)$ populations. 

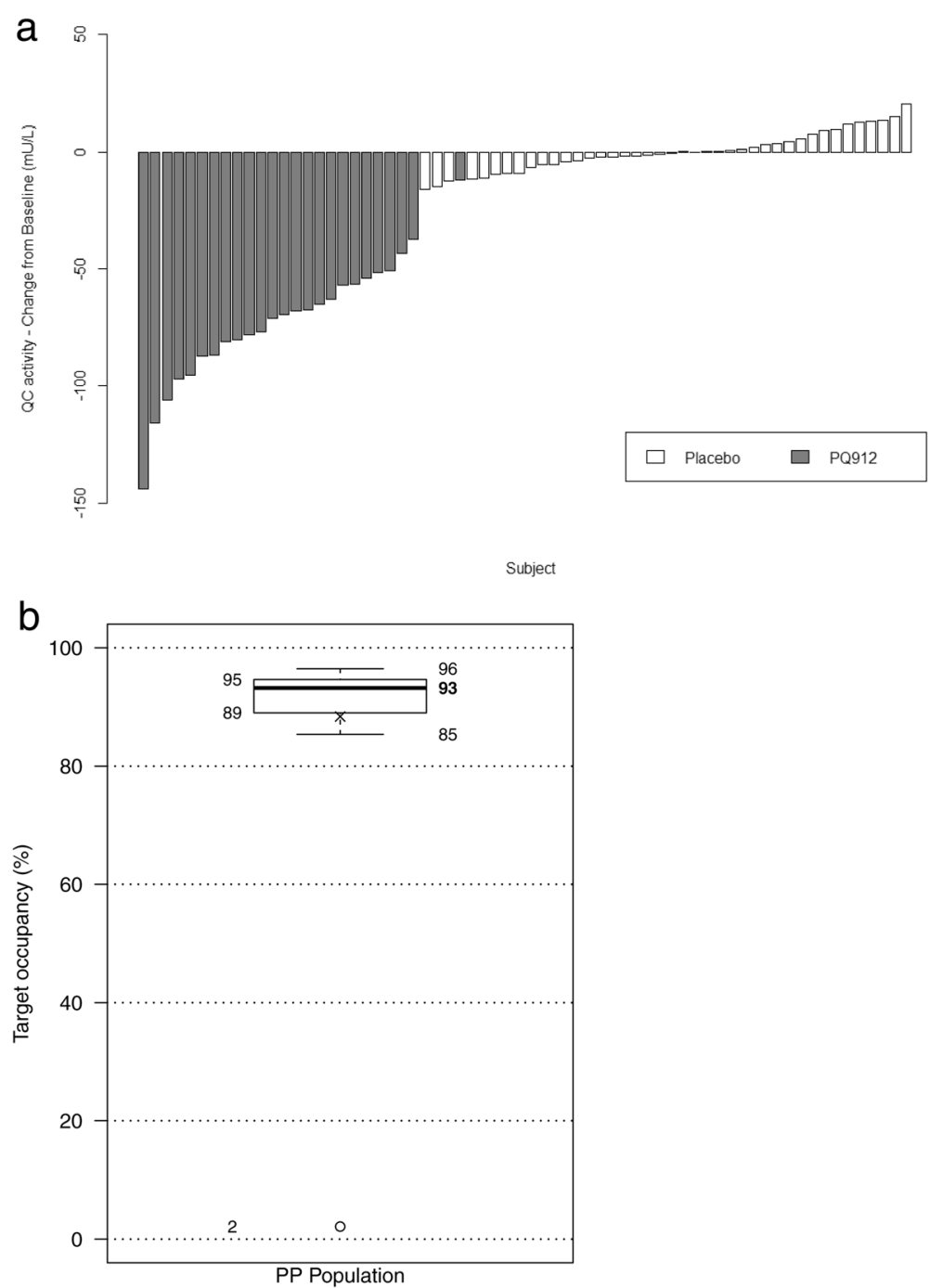

Fig. 4 a Glutaminyl cyclase activity ( $\mathrm{mU} / \mathrm{L}$ ): change from baseline at end of treatment. Per protocol population. b In-vivo target occupancy calculated from PQ912 levels. PQ912 treated, per protocol (PP) population

\section{Cognitive endpoints}

With regard to the individual cognitive test scores, we observed a PQ912 treatment effect on the One Back test $(P=0.05, d=0.23$, ITT population). The One Back test scores for subjects in the PQ912 group were slightly better (i.e., faster in response) compared to subjects in the placebo group. The mITT and PP analyses showed similar treatment effect sizes but these were not statistically significant. For the Detection test we found a treatment effect size of $d=0.21$ (ITT population), but this was not significant. We found no PQ912 treatment effects on the composite scores of episodic memory, executive function, attention and overall cognition.

We found no differences in Mini Mental State Examination (MMSE) scores between the study groups.
Analysis of covariance (ANCOVA) analyses for cognitive endpoints identified no effects of gender, ApoE, country or age.

\section{Discussion}

In this study we evaluated a novel therapeutic approach targeting the reduction of synaptotoxic and neurotoxic pGlu-A $\beta$ via inhibition of the enzyme glutaminyl cyclase by treating 120 subjects with either PQ912 or placebo.

The purpose of the study was-by using a high dose of PQ912 resulting in a high QC occupancy-to optimize the chance to find both early-on safety and tolerability signs and any signal on various sensitive exploratory outcome measures in a relatively short time frame of 12 weeks to inform the design of a comprehensive phase $2 b$ study. The selected outcome measures were tailored to the 
pathobiology of early AD with an emphasis on synaptic functional integrity (taking concurrently pharmacodynamic parameters related to the mechanism of action of PQ912 into account). Consequently, we introduced EEG recordings to capture potential effects on synaptic activity/integrity that directly relate to synaptic activity [19, 20]. In addition, level of neurogranin, a postsynaptic marker shown to be enhanced in AD [21], was measured. Both parameters are regarded as a centerpiece of pathology-related pharmacodynamic effects. As synaptic impairment directly results in cognitive memory deficits, the selection of sensitive cognitive tests in the NTB were used as efficacy readouts to test the hypothesis.

In a previously conducted large phase 1 study in healthy volunteers, PQ912 was considered safe and very well tolerated with no safety and tolerance signals identified. Therefore, the primary aim of this first in-patient study was to define the maximal tolerated dose of PQ912 and to identify the safety signal(s) associated with the MTD as proposed by the EMA guidelines for the development of new medicines for $\mathrm{AD}$ (Guideline on the clinical investigation of medicines for the treatment of Alzheimer's disease, 22 February 2018 http://www.ema.europa.eu/docs/en_GB/document_library/ Scientific_guideline/2018/02/WC500244609.pdf). To achieve this objective the highest multiple dose applied in the phase 1 study of $800 \mathrm{mg}$ bid was used, achieving more than $90 \%$ QC inhibition in CSF [13]. The second aim was to evaluate exploratory but converging readouts for early signals of efficacy after the relatively short treatment period. Another aim of the study was to confirm the PK/PD correlation previously established in healthy volunteers, in the target population of early AD.

The safety and tolerability profile of $800 \mathrm{mg}$ bid PQ912 as seen in this study indicate that the MTD has been reached. The results show an acceptable safety and tolerability profile with a comparable number of subjects reporting TEAEs or SAEs. However, more subjects treated with PQ912 discontinued treatment due to AEs specifically in the first 8 weeks of the exposure, with adverse events of the skin and subcutaneous organ system accounting for about half of the discontinuations. The types of skin adverse events were heterogeneous (reddening of skin/exanthema, rash, urticaria) and appeared 8-52 days after the start of treatment. There is currently no clear explanation for this observation, but similar skin reactions have been seen with other therapeutics (anti-epileptics, antibiotics, HIV drugs) suggesting that these events observed with PQ912 are caused by a hypersensitivity reaction to the drug or one of its metabolites; however, no data confirming such a hypothesis are currently available. As with other compounds inducing drug hypersensitivity, a slower titration schedule at the start of the treatment may allow for the adaptation of the immune system to the drug and reduce the number and severity of adverse events substantially. A slow titration regimen starting even with subpharmacological doses together with overall lower doses but still reaching a target occupancy on average of above 50\% (see Fig. 6) should improve adherence to treatment in future studies. The higher incidence of gastrointestinal and skin and subcutaneous tissue disorder-related TEAEs in the PQ912 group compared to the incidence observed in the phase 1 study in healthy elderly subjects is probably due to a longer treatment duration (12 weeks vs 10 days). As in the latter phase 1 study, we confirmed a $90 \%$ TO in the patients in the current study, underlying the validity of the previously defined PK/PD model [13]. Of particular relevance is that there was no higher incidence or specific adverse event pattern observed in the central nervous system organ class in the PQ912 group compared to placebo, which could at least indirectly affect cognitive performance negatively.

The study also evaluated a potential treatment effect on selected physiological substrates that, in contrast to glutamate as in 3-A $\beta$, carry $\mathrm{N}$-terminal glutamine, which is cyclized by $\mathrm{QC}$ as part of the maturation process of certain peptides and proteins. We chose the TRH and $\mathrm{GnRH}$ substrates as they have a high turnover, measured indirectly by quantifying the levels of TSH, T3 and T4 for the hypothalamic-pituitary-thyroid axis and testosterone for the hypothalamic-pituitary-gonadal axis. The finding that these parameters were not changed indicates also for patients that there is a high degree of on-target selectivity (glutamate vs glutamine), which has been shown in phase 1 and in animal safety studies $[9,13]$.

As regards exploratory biomarkers, the study revealed important pathology-related signals. There is now agreement that clinical pathological models of AD should be extended to include the effects of chronic inflammation. Consistent with this hypothesis [22, 23], YKL-40, a marker of astrocyte activation, has been shown to be increased in subjects with $\mathrm{AD}$ and $\mathrm{MCI}$ due to $\mathrm{AD}$, compared to matched controls [21]. In this study, PQ912 significantly reduced YKL-40 by approximately $5 \%$ of baseline level, which was not observed in the placebo group within 12 weeks. The effect of QC inhibition might relate to pGlu-A $\beta$ activating astrocytes in $A D$ [24]. The absolute reduction is small but, in relation to the scope of increase reported to be $10 \%$ (in MCI due to AD) to $25 \%$ (in $\mathrm{AD}$ ) compared to controls [25], the relative reduction amounts to $20-50 \%$ toward normal which is believed to be biologically meaningful.

The synaptic biomarker neurogranin was shown to be increased in $\mathrm{AD}$ compared to cognitively normal age-matched controls [21, 25]. Treatment with PQ912 reduced the neurogranin absolute level by approximately $4 \%$ in the PP population, although this decrease was not large enough to reach statistical significance. Similar to YKL-40, we consider the $4 \%$ decrease to be biologically meaningful considering that neurogranin is increased by about $35 \%$ at baseline in early $\mathrm{AD}$ disease versus healthy controls [25], thus in relative terms neurogranin was 
normalized by about $10 \%$ within the 3 -month treatment period which might be very meaningful. Furthermore, a recent phase 3 study in prodromal AD observed that treatment with the $A \beta$ antibody gantenerumab reduced CSF levels of neurogranin by about 10\% after 104 weeks of treatment at the highest dose used and this change was classified as a clear indication of a reduction in synaptic dysfunction early in the disease [26]. The effects observed on YKL-40 and neurogranin levels are viewed as warranted to include these exploratory biomarkers in a future phase 2/POC study.

In the context of the concept of PQ912 inhibiting synaptic toxicity of pGlu-A $\beta$ and an early $A D$ population, EEG analysis may be particularly important as a sensitive index of synaptic impairment $[19,20]$ because an increase in relative theta power is regarded as the most sensitive oscillatory activity marker in the earliest stages of $\mathrm{AD}$ [27]. In the few previous EEG studies with approved symptomatic $\mathrm{AD}$ drugs, changes in spectral power measures toward normal values, including decreases in relative theta power, have been reported with rivastigmine and donepezil treatment [28]. In the current study, PQ912 treatment showed a significant positive effect on EEG oscillatory activity compared to placebo. More specifically, global relative theta power decreased in the PQ912 group, whereas it increased in the placebo group (i.e., consistent with disease progression). The significant difference in EEG theta power observed in this study with a small to moderate effect size is regarded as a pharmacodynamically important indicator and is considered a very suitable noninvasive biomarker for further proof-of-concept studies of PQ912. Importantly, each EEG recording was carefully monitored by qualified technicians in order to avoid drowsiness and artifacts, and central quality check procedures shortly following each EEG recording allowed a repeat recording when suboptimal data were obtained. Using these standardized procedures, we ascertained the use of artifact-free EEG data in the alert but relaxed state and an unlikely influence of these factors in the results. However, future studies are necessary to evaluate a true diseasemodifying effect of PQ912 on synaptic function by demonstrating persistent differences in theta power after the cessation of the treatment period. Further, the analyses of the higher level functional connectivity using the phase lag index (PLI) and network topology measures, based on the minimum spanning tree of the full network, did not show differences between groups. As we would expect that changes in oscillatory activity, as reflected by relative power measures, are an expression of changes in functional connectivity between brain regions, we speculate that our selected measures were insensitive to the small effects of PQ912. The PLI and MST measures are highly 'pure' measures that were developed to exclude biases caused by volume conduction and differences in network sizes, respectively, but may have lost some relevant information due to these stringent calculation methods. We are now exploring the EEG dataset with other possibly more sensitive indices of functional connectivity [16, 29-31].

Analyses of RSfMRI parameters and use of eigenvector centrality mapping in clinical trials is in the experimental stage and there is no literature about regional differences in eigenvector centrality between groups of $\mathrm{AD}$ patients. The meaning of the statistically significant effect of PQ912 compared to placebo in the mean eigenvector centrality of the cuneus and lateral occipital regions (posterior) (effect size $d=0.58$ observed for this difference) therefore remains unclear. Furthermore, in a previously published patient study, an increase in this parameter was observed in $\mathrm{AD}$ subjects compared to healthy controls [32]. A whole-brain analysis of voxelwise eigenvector centrality in the treated versus placebo groups was different compared to the $\mathrm{AD}$ patients in the previously published study, putting into question whether these characteristics of sampling locations can be used to measure treatment effect in $\mathrm{AD}$. These regions did not have the same effect as in a study in AD subjects only. We therefore conclude that these sampling locations cannot be used to measure the treatment effect in AD.

Although it was not expected to see clinically relevant changes in the complete NTB in a 12-week time periodexpectation based on natural history cohorts and placebo arms of studies in a comparable early AD population (Cogstate normative data on file; Cogstate, New Haven, CT, USA)-we were interested to see whether the sensitive individual measures would move as an expression of synaptic recovery. In this context, it is noteworthy that an improvement in working memory was observed following treatment with PQ912. This improvement was accompanied by an improvement in alertness and, although moderate in magnitude, this treatment-related improvement was

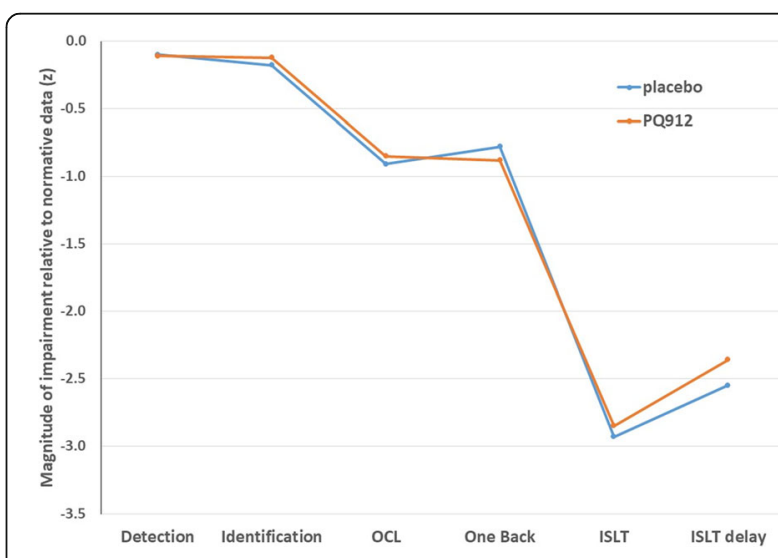

Fig. 5 Magnitude of impairment on individual neuropsychological tests at baseline assessment (Cogstate). Impairment defined as group mean performance expressed standardized score (z) using age-matched normative data (mean and SD). ISLT International Shopping List Task, OCL One Card Learning 
not large enough to reach statistical significance (effect size of both parameters: Cohen's $d>00.2$ ). Fig. 5 shows the degree of impairment at baseline of the placebo and PQ912 treatment groups for the individual cognitive tests when compared to Cogstate normative data for this early $\mathrm{AD}$ patient population (Cogstate normative data on file; Cogstate). The pattern of baseline impairment is as expected, with episodic memory being the most impaired area of function. The baseline deficit shown in the One Back Test, representing working memory, is a useful context for the treatment effect observed in this study (i.e., a 0.23 treatment effect in the context of a circa 0.7 deficit is a substantial return toward normal function). This might indicate early beneficial changes of PQ912 on cognition due to a more acute rescue effect on impaired synapses.

Regarding the exploratory readouts there are clear limitations in terms of treatment duration. One should note, however, that PQ912 has a new mechanism of action and one could not exclude it being reflected by a more acute effect on sensitive exploratory parameters as were selected for this study and which related to synaptic impairment. The results obtained are indeed giving important guidance for the design of the phase $2 \mathrm{~b}$ study.

To test for efficacy as a disease modifier, as we intend to do in a phase 2b program, it is of course necessary to show significant effects on slopes of disease progression which would need a longer treatment duration in an adequately powered trial. The primary endpoint of the phase $2 \mathrm{~b}$ program will be cognition. qEEG analysis will be incorporated as a secondary endpoint. As exploratory endpoints a set of biomarkers will be measured, including those biomarkers that showed a positive signal in this phase 2a study, such as neurogranin and YKL-40, and additionally a broader battery of CSF biomarkers (including synaptic proteins Contactin, Synaptosomal associated protein 25 and Growth associated protein 43; inflammatory markers sTREM2 and pGlu-CCL2; and markers of neurodegeneration NFL and VILIP1).

\section{Conclusions}

In summary, the goal of this study was reached as key guidance for the design of a proof-of-concept study was obtained. Firstly, we could clearly establish a maximum tolerated dose of PQ912 and identify skin and subcutaneous tissue as well as gastrointestinal disorders as safety signals associated with a high dose. This is aligned with recommendation of the EMA guideline to establish the MTD in phase 1 or early phase 2 . Secondly, the exploratory efficacy findings in this study are promising as the early signals found are all pointing in the same direction of improving function/inhibiting deterioration of synaptic activity and reducing neuroinflammation.

The study also achieved its objective to guide development of PQ912 in future studies in early AD using a lower dose range of PQ912. Animal studies have shown that a TO of about $60 \%$ revealed a very robust effect in terms of improving special learning and memory deficits in AD-like mice in concert with significant reductions in $\mathrm{pGlu}-\mathrm{A} \beta$, and therefore a range of $50-70 \%$ of target occupancy should be evaluated in future studies to assess the efficacy of PQ912 in early AD. From data obtained in the phase 1 MAD study with PQ912 in elderly healthy volunteers [12] it can be derived that a $60 \%$ TO was achieved with a dose of $200 \mathrm{mg}$ bid, thus with one quarter of the dose used in the SAPHIR study, and a dose of $300 \mathrm{mg}$ bid will lead to 70\% TO (Fig. 6) [13].

Lower doses and slower dose titration are highly likely to improve the safety and tolerance of PQ912-also because no relevant adverse events were seen in the first week of this study where all patients were on $400 \mathrm{mg}$ bid. A lower dose would still have a high degree of QC inhibition and thus it is expected to increase the benefit-risk ratio. The therapeutic concept of QC inhibition has the potential to improve synaptic functioning by reducing synaptotoxic effects of pGlu-A $\beta$ and long-term treatment may lead to a disease-modifying effect

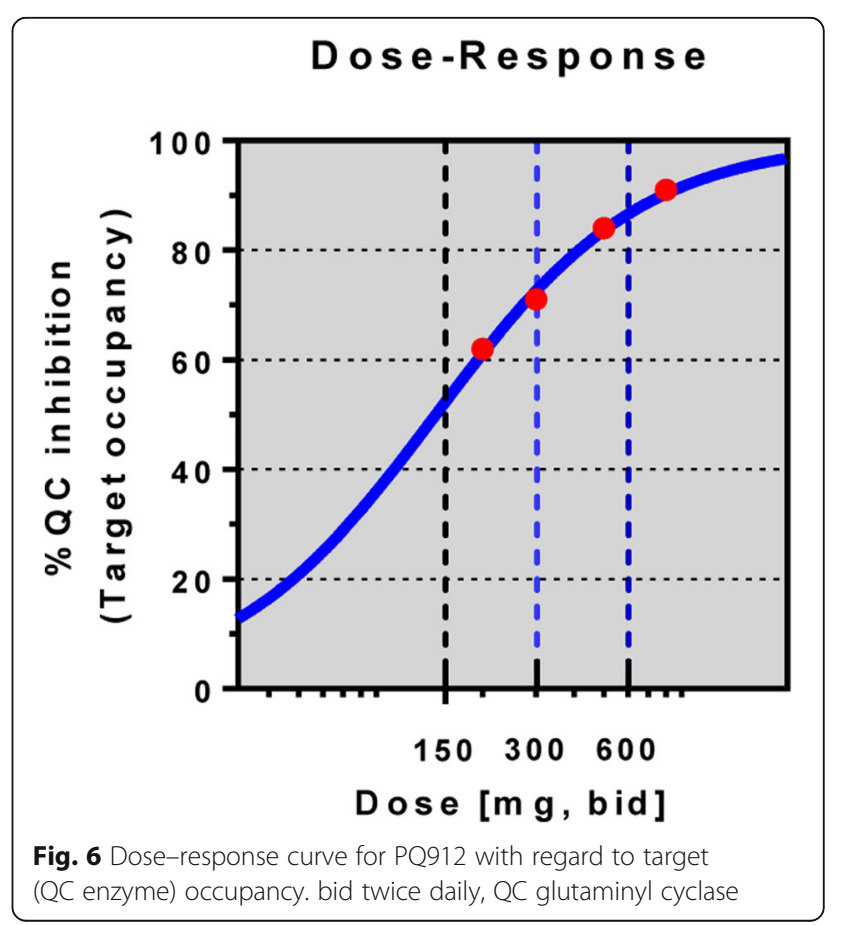


additionally supported by a reduction in neuroinflammation frequently associated with $\mathrm{AD}$.

\section{Additional files}

\section{Additional file 1: Extended methods. (DOCX $144 \mathrm{~kb})$}

Additional file 2: Table S1. Summary statistics and treatment effects of CSF, EEG, (RSf)MRI and cognition parameters. Difference (delta) plus 95\% $\mathrm{Cl}$ between treatment groups at EOT, controlled for baseline, age, gender, ApoE and country. Negative Cohen's D indicates lower values at EOT in PQ912 group (less increase or more decrease). (PDF 89 kb)

\section{Abbreviations}

AD: Alzheimer's disease; AE: Adverse event; ANCOVA: Analysis of covariance ApoE: Apolipoprotein E; AB: Amyloid beta; bid: Twice daily (bis in die); CFT: Category Fluency Test; Cl: Confidence interval; CSF: Cerebrospinal fluid; CTCAE: Common terminology criteria for adverse events; DMN: Default mode network; ECG: Electrocardiogram; EEG: Electroencephalography; EMA: European Medicines Agency; EOT: End of treatment; GnRH: Gonadotropinreleasing hormone; ISLT: International Shopping List Task; ITT: Intention to treat; $K_{\text {i: }}$ Inhibitory constant; LFT: Letter Fluency Test; MAD: Multiple ascending dose; MCl: Mild cognitive impairment; mITT: Modified intention to treat; MMSE: Mini Mental State Examination; MST: Minimum spanning tree; MTD: Maximum tolerated dose; NTB: Neuropsychological Test Battery; OCL: One Card Learning; PD/PK: Pharmacodynamics/pharmacokinetics; pGlu: Pyroglutamate; PLI: Phase lag index; PP: Per protocol; P-tau: Phosphorylated tau; QC: Glutaminyl cyclase; RD: Risk difference; RR: Relative risk; RSfMRI: Resting state functional magnetic resonance imaging; SAE: Serious adverse event; SD: Standard deviation; SOC: System organ class; T3: Triiodothyronine; T4: Prohormone thyroxine; TEAE: Treatment emergent adverse event; TO: Target occupancy; TRH: Thyrotropin-releasing hormone; TSH: Thyroid-stimulating hormone; YKL-40: Chitinase-3-like protein 1

\section{Acknowledgements}

The authors thank the patients and their family members participating in this SAPHIR study, as well as the investigators and their teams conducting this study. Medical writing support, under direction of the authors, was provided by A. Winsemius at Author!, Netherlands and was funded by Probiodrug. The authors thank T. Hoffman and A. Meyer for their support in coordinating the biomarker work and L. Smets for managing the collected data in the study. PS is co-editor-in-chief of Alzheimer's Research and Therapy but had no role whatsoever in handling this manuscript.

\section{Funding}

This study was funded by Probiodrug.

\section{Availability of data and materials}

The data and code that support the findings of this study are in SAS format and available upon reasonable request (contact person:

suzanne.bruins@probiodrug.de).

\section{Authors' contributions}

PS provided overall project supervision, and conceived and directed the study. NDP participated in the design and oversight of the trial, recruited patients and collected clinical data. MH, TG and TD recruited patients and collected the clinical data. AAG performed EEG analysis and provided intellectual input on EEG. AMW performed RSFMRI analysis and provided intellectual input on RSfMRI. CET led analysis of most of the CSF/biomarker assays. PM and JH provided intellectual input on cognitive testing. CMvB led the statistical analysis. IL provided conceptual scientific input and, jointly with SB, directed the operational aspects of the project. All authors edited the manuscript for content. All authors read and approved the final manuscript.

\section{Ethics approval and consent to participate}

Twenty-one centers in Europe (Belgium, Finland, France, Germany, Spain, Sweden and The Netherlands) participated in the study. The independent ethic committees at each site approved the protocol and amendments and the informed-consent documentation. All subjects and their caregiver provided written informed consent to participate in the study. The study was conducted in compliance with the declaration of Helsinki and in compliance with all International Conference on Harmonization Good Clinical Practice Guidelines.

\section{Consent for publication}

All authors approved the final version of the manuscript for submission.

\section{Competing interests}

PS has received consultancy/speaker fees (paid to the institution) from Eli-Lilly, GE Healthcare, Novartis, Probiodrug, Biogen, Roche, TauRx and EIP Pharma; he holds no stocks or options in Probiodrug. TG reported as an investigator and the national coordinating investigator for Germany in this trial; outside the submitted work he received consulting fees from Actelion, Eli Lilly, MSD, Novartis, Quintiles and Roche Pharma, lecture fees from Biogen, Lilly, Parexel and Roche Pharma, and grants to his institution from Actelion and PreDemTech. AAG received research support from Probiodrug and received research support from Boehringer Ingelheim via the VUmc Alzheimer Center. CET has functioned on advisory boards of Fujirebio and Roche, received nonfinancial support in the form of research consumables from ADxNeurosciences and Euroimmun, and performed contract research or received grants from Janssen Prevention Center, Boehringer, Brainsonline, AxonNeurosciences, EIP farma and Roche which are all unrelated to the present work. JH has in the past 2 years received honoraria and/or consulting fees from 23andMe, AbbVie, Access to Quality, AlzCure, Amgen, Anavex, Astellas Pharma, AstraZeneca, Avonex, Avraham, Axon, Axovant, Biogen Idec, Boehringer Ingelheim, Bracket, C4X Discovery, Cambridge Brain Sciences, Catenion, Cognitive Therapeutics, Compass Pathways, DeNDRoN, Enzymotec, Eisai, Eli Lilly, Forum Pharma, Fresh Forward, GfHEu, Heptares, Janssen Al, Johnson \& Johnson, Kaasa Health, Kyowa Hakko Kirin, LivaNova PLC, Lundbeck, MedAvante, Merck, MyCognition, Neurocog, Neurotrack, Novartis, Nutricia, Pfizer, Prana Biotech, Probiodrug, Prophase, ProStrakan, Regeneron, Reviva, Roche, Sanofi, Servier, Shire, Takeda, TCG, TransTech Pharma and Velacor. IL is an employee and shareholder of Probiodrug. NDP serves on the advisory board of Boehringer Ingelheim and Probiodrug, and is a member of the DSMB of Abbvie's M15-566 trial; he has received consultancy or speaker fees from Sanofi, Takeda, Janssen and Novartis; and he is CEO and co-owner of the Brain Research Center, Amsterdam. The remaining authors declare that they have no conflicts of interest.

\section{Publisher's Note}

Springer Nature remains neutral with regard to jurisdictional claims in published maps and institutional affiliations.

\section{Author details}

${ }^{1}$ Alzheimer Center and Department of Neurology, Amsterdam Neuroscience, VU University Medical Center, Amsterdam, The Netherlands. ${ }^{2}$ University of Eastern Finland, Institute of Clinical Medicine, Kuopio, Finland. ${ }^{3}$ Department of Psychiatry and Psychotherapy, Klinikum rechts der Isar, Technische Universität München, Munich, Germany. ${ }^{4}$ Department of Neurology, University of Münster, Münster, Germany. ${ }^{5}$ Department of Clinical Neurophysiology and MEG Center, Amsterdam Neuroscience, VU University Medical Center, Amsterdam, The Netherlands. ${ }^{6}$ Department of Clinical Chemistry, Neurochemistry Laboratory and Biobank, Amsterdam Neuroscience, VU University Medical Center, Amsterdam, The Netherlands. ${ }^{7}$ Department of Radiology, Nuclear Medicine and PET Research, Amsterdam Neuroscience, VU University Medical Center, Amsterdam, The Netherlands. ${ }^{8}$ Cogstate Ltd, Melbourne, Australia. Institute of Psychiatry, Psychology and Neuroscience, King's College London, London, UK. ${ }^{10}$ Julius Center for Health Sciences and Primary Care, UMC Utrecht, Utrecht, The Netherlands.

${ }^{11}$ Probiodrug AG, Halle, Germany. ${ }^{12}$ Brain Research Center, Amsterdam, The Netherlands.

Received: 15 May 2018 Accepted: 10 September 2018

Published online: 12 October 2018

\section{References}

1. Alzheimer's Association. 2015 Alzheimer's disease facts and figures. Alzheimers Dement. 2015;11:332.

2. Bloom GS. Amyloid- $\beta$ and tau: the trigger and bullet in Alzheimer disease pathogenesis. JAMA Neurol. 2014;71:505-8.

3. Ittner LM, Götz J. Amyloid- $\beta$ and tau-a toxic pas de deux in Alzheimer's disease. Nat Rev Neurosci. 2011;12:65-72. 
4. Jack CR Jr, et al. Hypothetical model of dynamic biomarkers of the Alzheimer's pathological cascade. Lancet Neurol. 2010;9:119-28.

5. Villemagne $V L$, et al. Amyloid $\beta$ deposition, neurodegeneration, and cognitive decline in sporadic Alzheimer's disease: a prospective cohort study. Lancet Neurol. 2013;12:357-67.

6. Ferreira ST, Lourenco MV, Oliveira MM, De Felice FG. Soluble amyloid- $\beta$ oligomers as synaptotoxins leading to cognitive impairment in Alzheimer's disease. Front Cell Neurosci. 2015:9:1-17.

7. Selkoe DJ. Alzheimer's disease is a synaptic failure. Science. 2002;298:789-91.

8. Nussbaum JM, et al. Prion-like behaviour and tau-dependent cytotoxicity of pyroglutamylated amyloid- $\beta$. Nature. 2012;485:651-5.

9. Hoffmann T, et al. Glutaminyl cyclase inhibitor PQ912 improves cognition in mouse models of Alzheimer's disease_-studies on relation to effective target occupancy. J Pharmacol Exp Ther. 2017;362:119-30.

10. Gilman AL, et al. Activity and architecture of pyroglutamate-modified amyloid- $\beta$ (AßpE3 42) pores. J Phys Chem B. 2014;118:7335-44.

11. Matos JO, Goldblatt G, Jeon J, Chen B, Tatulian SA. Pyroglutamylated amyloid- $\beta$ peptide reverses cross $\beta$-sheets by a prion-like mechanism. J Phys Chem B. 2014;118:5637-43.

12. Grochowska KM, et al. Posttranslational modification impact on the mechanism by which amyloid- $\beta$ induces synaptic dysfunction. EMBO Rep. 2017;18:1-20.

13. Lues I, et al. A phase 1 study to evaluate the safety and pharmacokinetics of PQ912, a glutaminyl cyclase inhibitor, in healthy subjects. Alzheimers Dement. 2015;1:182-95.

14. Albert MS, et al. The diagnosis of mild cognitive impairment due to Alzheimer's disease: recommendations from the National Institute on Aging-Alzheimer's Association workgroups on diagnostic guidelines for Alzheimer's disease. Alzheimers Dement. 2011;7:270-9.

15. McKhann GM, et al. The diagnosis of dementia due to Alzheimer's disease: recommendations from the National Institute on Aging-Alzheimer's Association workgroups on diagnostic guidelines for Alzheimer's disease. Alzheimers Dement. 2011;7:263-9.

16. Stam CJ, Nolte G, Daffertshofer A. Phase lag index: assessment of functional connectivity from multi channel EEG and MEG with diminished bias from common sources. Hum Brain Mapp. 2007;28:1178-93.

17. Stam CJ, et al. The trees and the forest: characterization of complex brain networks with minimum spanning trees. Int J Psychophysiol. 2014;92:129-38.

18. Tewarie $P$, van Dellen E, Hillebrand A, Stam CJ. The minimum spanning tree: an unbiased method for brain network analysis. Neurolmage. 2015;104:177-88.

19. Jeong J. EEG dynamics in patients with Alzheimer's disease. Clin Neurophysiol. 2004;115:1490-505

20. De Waal H, et al. Young Alzheimer patients show distinct regional changes of oscillatory brain dynamics. Neurobiol Aging. 2012;33:1008.e25-31.

21. Olsson B, et al. CSF and blood biomarkers for the diagnosis of Alzheimer's disease: a systematic review and meta-analysis. Lancet Neurol. 2016;15:673-84.

22. Heneka MT, et al. Neuroinflammation in Alzheimer's disease. Lancet Neurol. 2015;14:388-405.

23. Hellwig $\mathrm{K}$, et al. Neurogranin and YKL-40: independent markers of synaptic degeneration and neuroinflammation in Alzheimer's disease. Alzheimers Res Ther. 2015;7:74.

24. Hartlage-Rübsamen $M$, et al. Isoglutaminyl cyclase contributes to CCL2driven neuroinflammation in Alzheimer's disease. Acta Neuropathol. 2015; 129:565-83.

25. Janelidze $\mathrm{S}$, et al. Cerebrospinal fluid neurogranin and YKL-40 as biomarkers of Alzheimer's disease. Ann Clin Transl Neurol. 2015;3:12-20.

26. Blennow K, et al. Gantenerumab treatment reduces biomarkers of neuronal and synaptic degeneration in Alzheimer's disease. Alzheimers Dement. 2016; 12(Suppl):198.

27. Gouw AA, et al. EEG spectral analysis as a putative early prognostic biomarker in nondemented, amyloid positive subjects. Neurobiol Aging. 2017:57:133-42.

28. Rodriguez G, et al. Quantitative EEG changes in Alzheimer patients during long-term donepezil therapy. Neuropsychobiology. 2002:46:49-56.

29. Adler G, Brassen S, Jajcevic A. EEG coherence in Alzheimer's dementia. J Neural Transm (Vienna). 2003;110(9):1051-8.
30. Bruns A, Eckhorn R, Jokeit H, Ebner A. Amplitude envelope correlation detects coupling among incoherent brain signals. Neuroreport. 2000;11(7): 1509-14.

31. Pereda E, Quiroga RQ, Bhattacharya J. Nonlinear multivariate analysis of neurophysiological signals. Prog Neurobiol. 2005;77(1-2):1-37.

32. Binnewijzend MA, et al. Brain network alterations in Alzheimer's disease measured by eigenvector centrality in $\mathrm{FMRI}$ are related to cognition and CSF biomarkers. Hum Brain Mapp. 2014;35:2383-93.

\section{Ready to submit your research? Choose BMC and benefit from:}

- fast, convenient online submission

- thorough peer review by experienced researchers in your field

- rapid publication on acceptance

- support for research data, including large and complex data types

- gold Open Access which fosters wider collaboration and increased citations

- maximum visibility for your research: over $100 \mathrm{M}$ website views per year

At $\mathrm{BMC}$, research is always in progress.

Learn more biomedcentral.com/submissions 